\title{
Apparatus contribution to observed nonclassicality
}

\author{
Alfredo Luis (1) and Laura Ares (1) \\ Departamento de Óptica, Facultad de Ciencias Físicas, Universidad Complutense, 28040 Madrid, Spain
}

(Received 26 March 2020; accepted 13 July 2020; published 21 August 2020)

\begin{abstract}
Any nonclassical effect noticed in measured statistics is usually attributed just to the light state being observed. According to Born's rule, quantum probabilities are given by the overlap between the system state and measurement states in a quite symmetrical way. We demonstrate that the nonclassicality of the measurement is a necessary condition to obtain nonclassical statistics. To this end, we present a detector characterization for two-observable joint detection processes based on detector tomography. This is particularized to the most common signatures of nonclassical light.
\end{abstract}

DOI: 10.1103/PhysRevA.102.022222

\section{INTRODUCTION}

Experimental results that cannot be explained by classical electrodynamics are at the heart of quantum theory. These nonclassical effects, such as photon antibunching or quadrature squeezing, are always revealed by peculiarities of the observed statistics. Historically, these peculiarities have been attributed to the quantum character of the state being observed [1].

According to Born's rule, the probability of each measurement outcome is determined in a symmetrical way by the system state $|\psi\rangle$ and the measurement states $|m\rangle$, that is $p(m \mid \psi)=|\langle m \mid \psi\rangle|^{2}$, where typically $|m\rangle$ are the eigenvectors of the measured observable $M$. In more general terms, the measurement is described by a positive operator-valued measure (POVM) $\Delta(m)$ and the state by a density matrix $\rho$, so that $p(m \mid \rho)=\operatorname{tr}[\rho \Delta(m)]$.

The symmetry of Born's rule raises the question of whether the paradoxical experimental results might be ascribed to the measurement states as well. A quite remarkable example is the photoelectric effect as an observation of the light intensity. This is usually interpreted as proof of the quantum nature of the light, although it can be satisfactorily explained exclusively in terms of the quantum properties of the detector [2].

In this paper, we demonstrate that the nonclassicality of the detector is a necessary condition to obtain nonclassical statistics. This can be particularized to some simple and common signatures of nonclassical light such as sub-Poissonian statistics, quadrature squeezing, and photon anticorrelations. This question seems especially pertinent since typically the measurement states are highly nonclassical, say, number states and infinitely squeezed states, in photon-number and quadrature measurements, respectively.

The nonclassicality of states has been addressed in terms of quasiprobability distributions since the original works of Glauber and Surdashan [3,4]. Still today, the definition of nonclassical behavior rests on the Glauber-Surdashan $P(\alpha)$ representation $[5,6]$. It is the lack of a bona fide GlauberSurdashan $P(\alpha)$ distribution which defines a nonclassical state [1,7-9]. Moreover, this representation can be extended to every quantum operator [10], which makes it possible to define, in the same way, the measurement's nonclassicality as the lack of a bona fide Glauber-Surdashan $P(\alpha)$ distribution.

Based on this, we address whether quantum experiments admit a classical description of the measurement. We solve this in the negative, and nonclassicality of the detector is actually a necessary condition to observe any nonclassical property.

\section{NONCLASSICAL EVIDENCE}

Strictly speaking, a single-observable statistics cannot reveal by itself nonclassical behavior. This is because in classical physics we can always replicate any quantum probability distribution. The most clear quantum signature is the lack of a joint probability distribution for incompatible observables [11-14].

In the most general case, which is our starting point, the statistics can be properly represented in the system space by a positive operator-valued measure $\tilde{\Delta}(x, y)$,

$$
\tilde{p}(x, y \mid \rho)=\operatorname{tr}[\rho \tilde{\Delta}(x, y)],
$$

where $\rho$ is the density matrix of the system state, and $x, y$ are the outcomes in the measurement of two observables. We assume that the corresponding $x$ and $y$ marginals, which are $\tilde{\Delta}_{X}(x)=\int d y \tilde{\Delta}(x, y)$ and $\tilde{\Delta}_{Y}(y)=\int d x \tilde{\Delta}(x, y)$, provide complete information about two given system observables, say, $X$ and $Y$, respectively. This implies that, if $\Delta_{A}(a)$ are the exact, true POVMs corresponding to the system observables $A=X, Y$, there are functions $\mu_{A}\left(a, a^{\prime}\right)$ such that

$$
\Delta_{A}(a)=\int d a^{\prime} \mu_{A}\left(a, a^{\prime}\right) \tilde{\Delta}_{A}\left(a^{\prime}\right),
$$

for $a=x, y$. The functions $\mu_{A}\left(a, a^{\prime}\right)$ are state independent and completely known as far as we know for the measurement being performed.

The key idea is to extend this inversion (2) from the marginals to the complete joint distribution, in order to obtain an operator-valued measure $\Delta(x, y)[12,13,15-18]$,

$$
\Delta(x, y)=\int d x^{\prime} d y^{\prime} \mu_{X}\left(x, x^{\prime}\right) \mu_{Y}\left(y, y^{\prime}\right) \tilde{\Delta}\left(x^{\prime}, y^{\prime}\right),
$$


and the corresponding inferred joint distribution

$$
p(x, y \mid \rho)=\operatorname{tr}[\rho \Delta(x, y)] .
$$

In classical physics this program derives always in a bona fide joint probability distribution $[12,13]$. This is not the case in quantum physics, where $p(x, y \mid \rho)$ may not exist or take negative values as a clear signature of nonclassical behavior.

\section{APPARATUS CONTRIBUTION}

The main point of this paper reads that there must be intrinsic quantumness in any concrete experimental apparatus, say, in the behavior of the hardware itself, in order to be able to reveal any nonclassicality at all. This is that quantumness of the detector is a necessary condition to observe quantumness in the optical field, to the point that their mutual contributions can be hardly split.

Let us express this idea in two steps:

(i) Any nonclassical feature in the inferred $p(x, y \mid \rho)$ must have a clear correlate on a pathological feature of the inferred $\Delta(x, y)$. This is because $\rho$ is always a well-behaved density matrix. So if $p(x, y \mid \rho)$ does not exist, it is because $\Delta(x, y)$ does not exist, and if $p(x, y \mid \rho)$ takes negative values, it is because $\Delta(x, y)$ is not positive definite. Thus nonclassical phenomena require nonclassical detection.

(ii) The system state must be so that it reveals the pathological features already present in the detection scheme, say, the observed state must lie in the negative spectrum region of the detection POVM.

We elaborate on this to get to a useful complete characterization of nonclassical detectors, fully equivalent to that of nonclassical states. To this end we express the inferred operator measure (3) via the Glauber-Sudarshan $P$ representation as

$$
\Delta(x, y)=\frac{1}{\pi} \int d^{2} \alpha P(x, y \mid \alpha)|\alpha\rangle\langle\alpha|,
$$

where $|\alpha\rangle$ are the Glauber coherent states. The $P(x, y \mid \alpha)$ function provides a practical characterization of a detection process, in agreement with the idea of detector tomography [19-22]. In our context, it may serve as well to distinguish quantum from classical measurements. Clearly, if $\Delta(x, y)$ is pathological, then $P(x, y \mid \alpha)$ cannot be a classical-like bona fide probability distribution and the measurement is nonclassical. Vice versa, if the measurement is classical, the inferred joint statistics $p(x, y \mid \rho)$ is always a bona fide probability distribution that has no pathologies, as it can be clearly seen since after Eq. (5) the inferred joint distribution (4) becomes

$$
p(x, y \mid \rho)=\int d^{2} \alpha P(x, y \mid \alpha) Q(\alpha \mid \rho),
$$

with $Q(\alpha \mid \rho)$ the Husimi $Q$ function of $\rho$,

$$
Q(\alpha \mid \rho)=\frac{1}{\pi}\langle\alpha|\rho| \alpha\rangle .
$$

The key point here is that for every $\rho$, the function $Q(\alpha \mid \rho)$ exists and is non-negative, $Q(\alpha \mid \rho) \geqslant 0$. This demonstrates the first point of this paper, which is that for classical detectors we have always a classical interpretation. Equivalently, this means that there are nonclassical effects only provided that the measurement performed is itself nonclassical. Therefore the nonclassical criteria based on the $P$ function work for measurements as well as for states and serve as a suitable characterization of nonclassical detectors valid even beyond joint measurements.

We may ask what sorts of physical properties of a measurement apparatus correspond with the nonclassicality of the POVM used to characterize its statistical response to optical input states. We can say that these are exactly the same properties that correspond with the nonclassicality of optical input states. This is why we refer to the system-apparatus symmetry in the Born's rule.

This is after all a quite interesting feature of the quantum theory. Expressions such as $p(m \mid \psi)=|\langle m \mid \psi\rangle|^{2}$ or $p(m \mid \rho)=$ $\operatorname{tr}[\rho \Delta(m)]$ reveal that detection schemes are to be formally described in terms of light states, despite them all being made of matter. So we can ascribe to them quantum properties in exactly the same way we do to optical states.

\section{EXAMPLES}

After having linked nonclassicality with a pathological $P$ representation of the measurement $\Delta$ in a general enough framework, we can illustrate this scenario with some paradigmatic single-observable measurements through their $P$ function.

\section{A. Sub-Poissonian statistics}

A classic test of nonclassicality is sub-Poissonian statistics, $\Delta^{2} n<\langle\hat{n}\rangle$. For projection on number states $|n\rangle$ we have

$$
|n\rangle\left\langle n\left|=\int d^{2} \alpha p_{N}(n \mid \alpha)\right| \alpha\right\rangle\langle\alpha|,
$$

where $p_{N}(n \mid \alpha)$ is the Glauber-Sudarshan $P$ function of a number state [4],

$$
p_{N}(n \mid \alpha)=\frac{n ! e^{|\alpha|^{2}}}{2 \pi|\alpha|(2 n) !} \frac{\partial^{2 n}}{\partial|\alpha|^{2 n}} \delta(|\alpha|),
$$

which is extremely singular containing derivatives of the delta function. Let us examine whether the sub-Poissonian behavior still holds when we replace the above highly nonclassical $p_{N}(n \mid \alpha)$ by its closest classical version.

To this end we look for a POVM element $\Pi_{n}$ as close as possible to $|n\rangle\langle n|$ but with a well-defined positive and normalized $p_{N}(n \mid \alpha)$, i.e.,

$$
\Pi_{n}=\int d^{2} \alpha p_{N}(n \mid \alpha)|\alpha\rangle\langle\alpha| .
$$

As a criterion of closeness we may consider the maximum overlap between $\Pi_{n}$ and $|n\rangle\langle n|$, which is maximum $\left\langle n\left|\Pi_{n}\right| n\right\rangle$,

$$
\left\langle n\left|\Pi_{n}\right| n\right\rangle=\int d^{2} \alpha p_{N}(n \mid \alpha)|\langle n \mid \alpha\rangle|^{2} .
$$

It can be easily seen that $|\langle n \mid \alpha\rangle|^{2}$ as a function of $\alpha$ has a maximum for $|\alpha|^{2}=n$. Therefore the maximum overlap occurs when $p_{N}(n \mid \alpha)$ is concentrated at such a point, i.e.,

$$
p_{N}(n \mid \alpha)=\delta\left(n-|\alpha|^{2}\right),
$$




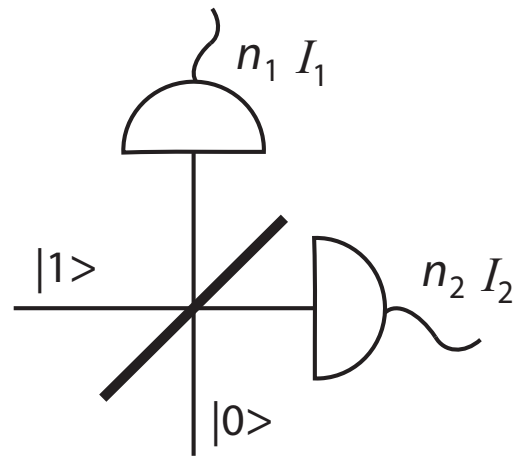

FIG. 1. Anticorrelation of photocounts for a single-photon input sate.

where $n$ is now a continuous variable, as already presented in Ref. [23]. In such a case the corresponding statistics reads

$$
p_{N}(n \mid \rho)=\frac{1}{2} \int_{2 \pi} d \phi Q\left(\alpha=\sqrt{n} e^{i \phi} \mid \rho\right),
$$

where we have used that $d^{2} \alpha=(1 / 2) d|\alpha|^{2} d \phi$ and $\phi$ is the phase of $\alpha$. This statistics can be related to the actual exact photon-number distribution in the form

$$
p_{N}(n \mid \rho)=\sum_{m=0}^{\infty} \frac{n^{m} e^{-n}}{m !}\langle m|\rho| m\rangle,
$$

where $|m\rangle$ are number states.

Let us compare the variance obtained from the classical versus the quantum detectors, with the following explicit form of classical $\left\langle n^{k}\right\rangle$ and quantum $\left\langle\hat{n}^{k}\right\rangle$ moments, for any integer $k$,

$$
\left\langle n^{k}\right\rangle=\int d^{2} \alpha n^{k} p_{N}(n \mid \rho), \quad\left\langle\hat{n}^{k}\right\rangle=\operatorname{tr}\left[\rho\left(a^{\dagger} a\right)^{k}\right] .
$$

Taking into account that

$$
\int_{0}^{\infty} d n n^{k} e^{-n}=k !
$$

we readily get from Eqs. (13) and (14),

$$
\langle n\rangle=\langle\hat{n}\rangle+1, \quad\left\langle n^{2}\right\rangle=\left\langle\hat{n}^{2}\right\rangle+3\langle\hat{n}\rangle+2,
$$

so that

$$
\Delta^{2} n=\left\langle n^{2}\right\rangle-\langle n\rangle^{2}=\Delta^{2} \hat{n}+\langle n\rangle \geqslant\langle n\rangle .
$$

So, if we replace quantum by classical-like measurement, all states are Poissonian or super-Poissonian. Therefore, we may safely say that sub-Poissonian statistics holds only if the measurement is sub-Poissonian itself, and so it is nonclassical.

\section{B. Anticoincidences}

Next, we move from sub-Poissonian statistics to the anticorrelation of photocounts in the typical scenario displayed in Fig. 1, as the flagship of quantum optics. A single photon impinges on a lossless beam splitter and two joint intensity measurements are performed at the outputs of the beam splitter. Since the photon is indivisible, the detectors can never trigger both simultaneously, so that $\left\langle\hat{n}_{1} \hat{n}_{2}\right\rangle=0$. This is maybe

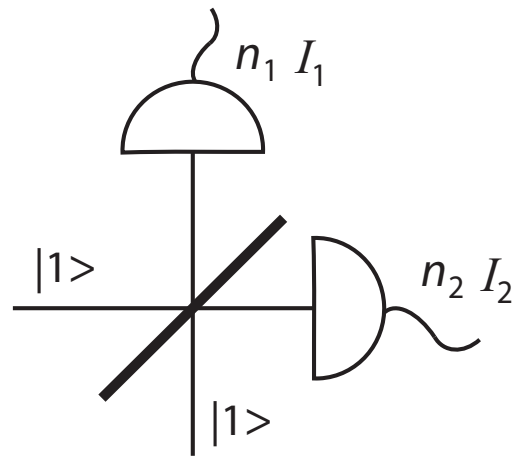

FIG. 2. Anticorrelation of photocounts for a twin-photon input sate.

the most clear and simple evidence of the quantum nature of light [24].

Thus we consider a two-mode version of the classical measurement described by the $P_{N}(n \mid \alpha)$ function in Eq. (12) to obtain the statistics when the field is in the one-photon state,

$$
p\left(n_{1}, n_{2} \mid \rho\right)=\left(R n_{1}+T n_{2}\right) e^{-n_{1}-n_{2}},
$$

where $R, T$ are the transmission and reflection coefficients with $T+R=1$. We simply get $\left\langle n_{1} n_{2}\right\rangle=2$, so that the alleged quantum effect would never be observed if the detectors were classical-like devices.

Along the same lines we may examine the Hong-OuMandel effect illustrated in Fig. 2 [25], where two photons impinge simultaneously on the input ports of a lossless $50 \%$ beam splitter. The quantum theory predicts the result $\left\langle\hat{n}_{1} \hat{n}_{2}\right\rangle=$ 0 again, as evidence of the quantum nature of light. However, this result is not preserved if we replace the detectors by classical-like measurements as before, since the joint statistics would be

$$
p\left(n_{1}, n_{2} \mid \rho\right)=\frac{1}{4}\left(n_{1}^{2}+n_{2}^{2}\right) e^{-n_{1}-n_{2}},
$$

leading to $\left\langle n_{1} n_{2}\right\rangle=3$.

\section{Quadrature squeezing}

As the last example we present quadrature squeezing $\Delta^{2} x<1 / 4$, where $x$ represents a field quadrature $x=\operatorname{Re}\{\alpha\}$, which is incompatible with a bona fide $P(\alpha \mid \rho)$. Here again, the statistics of a quadrature measurement results by projection on the quadrature eigenstates that are highly nonclassical being infinitely squeezed. We carry out the closest classical measurement by replacing the strongly quantum $p_{X}(x \mid \alpha)$ by

$$
p_{X}(x \mid \alpha)=\delta(x-\operatorname{Re}\{\alpha\}) .
$$

so that the corresponding statistics reads

$$
p_{X}(x \mid \rho)=\int_{-\infty}^{\infty} d y Q(\alpha=x+i y)
$$

that is,

$$
p_{X}(x \mid \rho)=\sqrt{\frac{2}{\pi}} \int_{-\infty}^{\infty} d x^{\prime} e^{-2\left(x-x^{\prime}\right)^{2}}\langle x|\rho| x\rangle,
$$


where $|x\rangle$ are quadrature eigenstates. Here again, computing the first two moments we get

$$
\langle x\rangle=\langle\hat{X}\rangle, \quad\left\langle x^{2}\right\rangle=\left\langle\hat{X}^{2}\right\rangle+\frac{1}{4},
$$

so that $\Delta^{2} x=\Delta^{2} \hat{X}+\frac{1}{4} \geqslant \frac{1}{4}$. Therefore, with classical quadrature measurements there would be no nonclassical behavior regarding this physical variable.

We utilize the latter example to illustrate that there are actually classical detectors in a quantum world. In order to do this, we show that a real detector with a classical description emerges from a quantum one after thermalization, in the sense of adding thermal noise to the quantum device. This is actually the same idea of combining a nonclassical state with thermal light to remove all its nonclassical properties [23,26]. To this end, we substitute each highly nonclassical element of the quadrature measurement, $\Pi_{X}=|x\rangle\langle x|$, for a combination of them with thermal distribution,

$$
\Pi_{X}^{\tau}=\int d^{2} \alpha \frac{e^{-|\alpha|^{2} / 2 \tau}}{2 \pi \tau} D^{\dagger}(\alpha)|x\rangle\langle x| D(\alpha),
$$

where $D(\alpha)$ is the displacement operator and $\tau$ is a measure of the amount of thermal fluctuations, leading to

$$
\Pi_{X}^{\tau}=\int d^{2} \alpha \frac{e^{-|\alpha|^{2} / 2 \tau}}{2 \pi \tau}\left|x+\frac{\operatorname{Re}(\alpha)}{2}\right\rangle\left\langle x+\frac{\operatorname{Re}(\alpha)}{2}\right| .
$$

The resulting statistics $\operatorname{tr}\left[\rho \Pi_{X}^{\tau}\right]$ is identical to (23) for $\tau=1$. Alternatively, the same result is obtained if we model a real detector as an ideal one, preceded by a beam splitter of finite transmittance, where $\tau$ would be proportional to the inverse of the quantum efficiency [27].

\section{Bell tests}

As the last example of nonclassical behavior we study the violation of Bell inequalities. In a recent work [28] we have proposed a simultaneous joint measurement of all observables involved in the Bell tests in order to solve the difficulties caused by measuring noncompatible observables. Applying then an inversion procedure of the kind in Eq. (3) to the recorded statistics, we concluded that the violation of Bell inequalities is equivalent to a retrieved joint distribution $p(x, y, u, v)$ taking negative values, in agreement with Fine's theorem [11]. Here, we show how any violation of Bell inequalities requires nonclassical detection.

We analyze a typical Bell test, with a pair of twodimensional subsystems or qubits, $A$ and $B$, measuring two dichotomic observables in each of them, say, $X, Y$ and $U, V$, respectively. The inferred joint distribution is [28]

$$
p(x, y, u, v \mid \rho)=\operatorname{tr}[\rho \Delta(x, y, u, v)],
$$

where

$$
\Delta(x, y, u, v)=\Delta(x, y) \otimes \Delta(u, v),
$$

with

$$
\begin{aligned}
& \Delta_{A}(x, y)=\frac{1}{4}\left[1+S_{A}(x, y) \cdot \boldsymbol{\sigma}\right], \\
& \Delta_{B}(u, v)=\frac{1}{4}\left[1+\boldsymbol{S}_{B}(u, v) \cdot \boldsymbol{\sigma}\right],
\end{aligned}
$$

where, for example, in the the simplest situation,

$$
\begin{aligned}
& \boldsymbol{S}_{A}(x, y)=x \boldsymbol{S}_{X}+y \boldsymbol{S}_{Y}, \\
& \boldsymbol{S}_{B}(u, v)=u \boldsymbol{S}_{U}+v \boldsymbol{S}_{V} .
\end{aligned}
$$

with $\sigma$ the three Pauli matrices, $x, y, u, v= \pm 1$, and $S_{W}, W=$ $X, Y, U, V$ are three-dimensional, real, unit vectors that specify the particular observables being measured as $\Delta_{W}(w)=$ $\left(1+w \boldsymbol{S}_{W} \cdot \boldsymbol{\sigma}\right) / 2, w=x, y, u, v$.

Then, $\Delta(x, y, u, v)$ will be positive and well behaved provided that both $\Delta_{A}(x, y)$ and $\Delta_{B}(u, v)$ are positive and well behaved, which holds provided that $\left|\boldsymbol{S}_{A}(x, y)\right| \leqslant 1$ and $\left|\boldsymbol{S}_{B}(u, v)\right| \leqslant 1$ for all $x, y, u, v$. In such a case, the measurement performed in both subsystems will admit a classicallike description. This situation can be well illustrated by considering the case of the singlet maximally entangled state which leads to [28]

$$
p(x, y, u, v)=\frac{1}{16}\left[1-S_{A}(x, y) \cdot S_{B}(u, v)\right],
$$

which is pathological provided that either $\left|\boldsymbol{S}_{A}(x, y)\right|>1$, or $\left|\boldsymbol{S}_{B}(u, v)\right|>1$, or both, that is, when either $\Delta_{A}(x, y)$, or $\Delta_{B}(u, v)$, or both, are nonclassical.

\section{CONCLUSIONS}

To conclude, we have used the phase-space representatives of measurements to characterize their classical or nonclassical behavior. In particular, we have presented a detector model for two-observable joint detection processes. This allowed us to link the classical or quantum nature of detectors with the nonclassical properties of the recorded and inferred statistics. With this we can demonstrate that the nonclassicality of the detector is a necessary condition to obtain nonclassical statistics. We have shown explicitly that this is the case in the most typical signatures of nonclassical behavior, such as subPoissonian statistics, quadrature squeezing, photon anticorrelations, and Bell violations, showing that they unavoidably require detectors that are themselves nonclassical. Then, we conclude that there is no quantum light without quantum detectors.

\section{ACKNOWLEDGMENTS}

L.A. and A.L. acknowledge financial support from Spanish Ministerio de Economía y Competitividad Project No. FIS2016-75199-P. L.A. acknowledges financial support from European Social Fund and the Spanish Ministerio de Ciencia Innovación y Universidades, Contract Grant No. BES-2017-081942.
[1] L. Mandel, Phys. Scr. T 12, 34 (1986).

[2] G. Greenstein and A. G. Zajonc, The Quantum Challenge: Modern Research on the Foundations of Quantum Mechanics (Jones \& Bartlett Learning, Burlington, MA, 2005).
[3] R. J. Glauber, Phys. Rev. 131, 2766 (1963).

[4] E. C. G. Sudarshan, Phys. Rev. Lett. 10, 277 (1963).

[5] J. Sperling and W. Vogel, Phys. Scr. 95, 034007 (2020).

[6] T. Kiesel, Phys. Rev. A 87, 062114 (2013). 
[7] U. M. Titulaer and R. J. Glauber, Phys. Rev. 140, B676 (1965).

[8] L. Mandel and E. Wolf, Optical Coherence and Quantum Optics (Cambridge University Press, Cambridge, U.K., 1995).

[9] M. O. Scully and M. S. Zubairy, Quantum Optics (Cambridge University Press, Cambridge, U.K., 1997).

[10] C. L. Mehta, Phys. Rev. Lett. 18, 752 (1967).

[11] A. Fine, Phys. Rev. Lett. 48, 291 (1982).

[12] A. Luis, Opt. Lett. 41, 1789 (2016).

[13] A. Luis and L. Monroy, Phys. Rev. A 96, 063802 (2017).

[14] C. Sanchidrian-Vaca and A. Luis, Phys. Rev. A 97, 043810 (2018).

[15] W. M. Muynck, Foundations of Quantum Mechanics, an Empiricist Approach (Kluwer Academic, Dordrecht, 2002).

[16] W. M. de Muynck and H. Martens, Phys. Rev. A 42, 5079 (1990).

[17] W. M. de Muynck, Phys. Lett. A 182, 201 (1993).

[18] W. M. de Muynck, J. Phys. A: Math. Gen. 31, 431 (1998).
[19] A. Luis and L. L. Sánchez-Soto, Phys. Rev. Lett. 83, 3573 (1999).

[20] A. Luis, Phys. Rev. A 62, 054302 (2000).

[21] J. S. Lundeen, A. Feito, H. Coldenstrodt-Ronge, K. L. Pregnell, Ch. Silberhorn, T. C. Ralph, J. Eisert, M. B. Plenio, and I. A. Walmsley, Nat. Phys. 5, 27 (2009).

[22] H. B. Coldenstrodt-Ronge, J. S. Lundeen, K. L. Pregnell, A. Feito, B. J. Smith, W. Mauerer, Ch. Silberhorn, J. Eisert, M. B. Plenio, and I. A. Walmsley, J. Mod. Opt. 56, 432 (2009).

[23] K. Kim, J. Korean Phys. Soc. 64, 155 (2014).

[24] P. Grangier, G. Roger, and A. Aspect, Europhys. Lett. 1, 173 (1986).

[25] C. K. Hong, Z. Y. Ou, and L. Mandel, Phys. Rev. Lett. 59, 2044 (1987).

[26] C. T. Lee, Phys. Rev. A 44, R2775 (1991).

[27] B. L. Schumaker, Opt. Lett. 9, 189 (1984).

[28] E. Masa, L. Ares, and A. Luis, Phys. Lett. A 384, 126416 (2020). 